\title{
“I Can't Get No Satisfaction": Measuring Student Satisfaction in the Age of a Consumerist Higher Education
}

\author{
Carl Senior *, Elisabeth Moores and Adrian P. Burgess \\ Department of Psychology, School of Life and Health Sciences, Aston University, Birmingham, United Kingdom
}

Keywords: student engagement, student satisfaction, data primacy, measurements, innovation, universities

One could be excused for failing to recognize today's universities as the inheritors of the global higher education system that arose more than 70 years ago from the ashes of the Second World War. A wave of post-war optimism ushered in a global movement with a utopian vision in which arbitrary divisions such as class, gender, and race would be transcended in the pursuit of academic enlightenment (Scott, 1995). Universities were to be one of the key drivers of this change. But, contemporary academia is a distinctly different beast. The enlightenment values of the liberal education model, once the dominant philosophy in universities across the world, are gradually being supplanted by a consumerist ideology (Furedi, 2011): Yesterday's "Cathedrals of learning" are being replaced by today's "Supermarkets of facts"

The rise of the consumer model of universities, derided by many, has brought distinct benefits that the enlightenment model failed to achieve. One could perhaps marvel at the fact that here is a single philosophy that has effectively transcended national boundaries. By advocating a consumerist philosophy, managers of Higher Education (HE) institutions have been able to employ the full gamut of market forces to drive innovation in their day-to-day practice (Christensen and Eyring, 2011). Not least of the achievements arising from this, has been the massive expansion of the franchise such that university education, once the prerogative of a small social elite who

Edited by:

Jesus de la Fuente, University of Almería, Spain

Reviewed by:

Georgina Randsley de Moura, University of Kent, United Kingdom

*Correspondence: Carl Senior c.senior@aston.ac.uk

Specialty section:

This article was submitted to Educational Psychology, a section of the journal Frontiers in Psychology

Received: 11 April 2017 Accepted: 29 May 2017 Published: 08 June 2017

Citation: Senior $C$, Moores $E$ and Burgess AP (2017) "I Can't Get No Satisfaction": Measuring Student Satisfaction in the Age of a Consumerist Higher

Education. Front. Psychol. 8:980. doi: 10.3389/fpsyg.2017.00980 valued learning for the sake of enlightenment, is now the expectation of a large proportion of the population whose primary desire is to improve their position on the subsequent employment market (Tomlinson, 2008). Today's universities have been quick to meet this need and institutional offerings have followed suit, enabling students to gain experience in a range of additional and subsidiary programmes that focus on the provision of "value added" benefits (Deane and Stanley, 2015). Here, students are encouraged to develop a wide range of transferable skills from entrepreneurship and enterprise to a knowledge of intellectual property rights and even leadership skills.

The embrace of the Business-to-Consumer model of HE also presents university managers with many challenges (See e.g., Deloitte's, 2015; "Making the grade" report). What does it mean to be a university in the modern consumerist era? How can the traditional values of scholarship and standards be preserved in a customer-focussed institution? How does the HE sector continue to enable graduates to become effective citizens who contribute to the betterment of society? Most important of all from the consumer model perspective, "What do students actually expect from $\mathrm{HE}$ and how are education providers framing and meeting these expectations?" The key metric for this last question is student satisfaction, yet, despite its almost ubiquitous position as a tool for university managers, the concept of "student satisfaction" remains ephemeral and surprisingly little is known about what makes a student satisfied with their experience of HE or how it can be measured effectively.

Only in the last 10 years or so has work emerged that has started to examine the institutional drivers of student satisfaction (Mai, 2005). Clemes and colleagues examined the

\footnotetext{
${ }^{1}$ Phrase attributed to the late Dr. Mike Harris of the University of Birmingham, UK.
} 
various relationships between a range of institutional factors and their relationship to satisfaction in the student cohort (Clemes et al., 2008). They found a significant relationship between satisfied students and the quality of the teaching with a mediating role for institutional reputation. A significant predictive relationship was also reported between satisfaction and intended future outcomes post-graduation. Alves and Raposo (2007) also examined the behaviors that effectively predicted student satisfaction and also revealed that the quality of teaching experience was a key driver. More surprisingly, they also found that institutional reputation was actually a more influential predictor of student satisfaction than teaching quality. So, it would seem that students are satisfied if they receive good teaching at a reputable institute.

Alves and Raposo (2007) went on to examine the effects of having a cohort of satisfied students. They found that satisfaction bred loyalty. Students who were satisfied were more loyal to the institution and were more likely to engage with alumni activities and maintain an ongoing relationship with their alma mater. As universities in many countries expend considerable effort and money on establishing a body of loyal graduates that may one day reward them with a financial return, this is clearly an important finding. Gibbons et al. (2015) show that NSS scores have a small but statistically significant effect on University applications at a subject level, but suggest that this effect is primarily driven by league table positions (rather than original data).

The measurement of student satisfaction is one that undoubtedly vexes institutional managers around the world because, despite its importance, measuring satisfaction is not trivial and presents a number of challenges (see e.g., Cashin, 1990). For example, how can the new and emerging expectations of students be measured in an effective fashion? How can data be collected in a timely manner to ensure that managers can effect improvement in the immediate learning environment? How can we encourage the free flow of information from the consumers to the managers and vice versa that is so important to maintaining success in the modern competitive HE environment? Within $\mathrm{HE}$, managers collect information on student satisfaction using a range of mechanisms designed to ensure that the expectations of the student are met at every stage of their progression through university. Timetabled one-to-one meetings between staff and students, drop-in sessions and staff-student consultative committees are now so pervasive that only the most insulated of academics can have failed to recognize the changing zeitgeist. Although these devices may be effective at the individual level, these strategies probably have little impact at the institutional level and almost none across the sector as a whole.

To address this problem, most developed countries use some form of national survey that they deliver to students to collect a range of measures of student satisfaction. Japanese academic managers make use of results from the Japanese College Student Survey (JCSS) and the Japanese Freshman Survey (JFS) both of which have been studied extensively (see e.g., Yamada, 2013). The National Survey for Student Engagement (NSSE) is used in the USA (Kuh, 2003). The Course Experience Questionnaire (CEQ) is employed in Australia (Ramsden, 1991; Wilson et al., 1997) and in the UK the National Student Survey (NSS: Richardson et al., 2007 ) is completed by almost 300,000 final year undergraduate students each year. In the UK, national league tables of the NSS results are published annually and are readily available to anyone contemplating applying to university. As such, NSS scores are an important driver of institutional change and woe betide the subject group or individual teacher who is perceived to be adversely affecting student ratings. Despite its influence, however, there is considerable debate as to whether the NSS offers sufficient discrimination between Universities to be useful, or measures fairly across different subject disciplines (Cheng and Marsh, 2010; Yorke et al., 2014).

The one consistent finding of all this work is that high quality teaching is an important factor in student satisfaction; a finding that should surprise no one. Excellence in teaching is the sine qua non of a modern university and the power of consumer choice alone is enough to ensure that a university which does not deliver its key product (effective teaching) to its consumer base (students) does not remain in business (see e.g., Mathooko and Ogutu, 2015; Milian et al., 2016). But why then is so much effort and $\operatorname{cost}^{2}$ dedicated to measuring aspects of student attitudes when the results are so clearly aligned with common sense?

One reason may be that the role of universities is changing. The rise of wide scale reforms across the global HE sector are inexorably driving University management away from the delivery of effective teaching toward the delivery of a more transferable and professional skillset that is more closely aligned to the graduate expectations of successful employment. On first consideration such a development may seem at odds with the traditional and clearly non-vocational model of a university which first emerged in the mid-nineteenth century with the early writings of Cardinal John Henry Newman ${ }^{3}$. Yet, even within this early philosophy there existed a clear advocacy for the development of skills acquired through general critical and reflective abilities that were applicable to any role in the workplace. In this model, university learning was less about employment and more about the ability to be successful in society, whereas in contemporary $\mathrm{HE}$ the development of a focused professional skillset has become an increasingly dominating influence. Indeed, in our view, it is likely that today's satisfied students are most likely to be those who have experienced a programme of study that aligns itself directly with their expectations for subsequent and very specific employment.

Such a shift is inevitable and, as we have previously argued, in order to deliver an effective learning experience the modern day university manager needs to embrace the full scope of the student activities that occur both on and off campus (Senior et al., 2014). This portfolio of experience should include the development of professional skills that they have acquired outside the classroom and in the world of work. However, as noted above this can be a vast and wide portfolio of professional skills (see also Bridges, 1993; Moores and Reddy, 2012; Reddy and Moores, 2012). Whilst institutions across the global HE sector are readily aligning the student experiences within the campus to meet these external

\footnotetext{
${ }^{2}$ In the UK student satisfaction is measured with the annual National student Survey (NSS) that costs $£ 2.4 \mathrm{M}$ to develop and deliver. Source: http://webarchive. nationalarchives.gov.uk/20120118164922/http://hefce.ac.uk/pubs/board/2004/93/ B39.pdf

${ }^{3}$ John Henry Newman, in his seminal essay "The Idea of a University" (1852) made a powerful and influential case for the liberal ideal of a university.
} 
expectations and deliver a truly engaged model of scholarship, they tend to lack the means to measure these activities and to ensure that modern day students are satisfied with the learning experience they receive (Van de Ven, 2007).

Upon reflection, we now make three recommendations for institutional managers and policy directors to consider. First, the academic environment has changed; managers can no longer expect students to be satisfied with excellent teaching alone. Students expect the provision of excellence with regards to professional skills that they can transfer to the post-graduation workforce and thereby harvest the economic and social benefits that attracted them to University study in the first place. Second, there needs to be a detailed and thorough statistical examination of the current means by which student satisfaction is measured across the HE sector. In our view, current measures of student satisfaction are no longer adequate in scope to meet the changing needs of students and the developing roles of universities. Third,

\section{REFERENCES}

Alves, H., and Raposo, M. (2007). Conceptual model of student satisfaction in higher education. Total Qual. Manag. 18, 571-588. doi: 10.1080/14783360601074315

Bridges, D. (1993). Transferable skills: a philosophical perspective. Stud. High. Educ. 18, 43-51. doi: 10.1080/03075079312331382448

Cashin, W. E. (1990). Students do rate different academic fields differently. New Dir. Teach. Learn. 43, 113-121. doi: 10.1002/tl.37219904310

Cheng, J. H., and Marsh, H. W. (2010). National Student Survey: are differences between universities and courses reliable and meaningful? Oxford Rev. Educ. 36, 693-712. doi: 10.1080/03054985.2010.491179

Christensen, C. M., and Eyring, H. J. (2011). The Innovative University: Changing the DNA of Higher Education from the Inside Out, 1st Edn. San Francisco, CA: Jossey-Bass.

Clemes, M. D., Gan, C. E., and Kao, T. H. (2008). University student satisfaction: an empirical analysis. J. Market. High. Educ. 17, 292-325. doi: 10.1080/08841240801912831

Deane, E. M., and Stanley, K. (2015). “The Student leadership in curriculum development and reform project," in Research and Development in Higher Education: Learning for Life and Work in a Complex World, Vol. 38, eds T. Thomas, E. Levin, P. Dawson, K. Fraser and R., Hadgraft (Melbourne, VIC), 130-140, (Accessed July 6-9).

Deloitte (2015). Making the Grade 2015: The Key Issues Facing the UK Higher Education Sector.

Furedi, F. (2011). "Introduction to the marketisation of higher education and the student as consumer," in The Marketisation of Higher Education and the Student as Consumer, eds M. Molesworth, M. Scullion, and R. Nixon (Oxford: Routledge), 1-9.

Gibbons, S., Neumayer, E., and Perkins, R. (2015). Student satisfaction, league tables and university applications: evidence from Britain. Econ. Educ. Rev. 48, 148-164. doi: 10.1016/j.econedurev.2015.07.002

Kuh, G. D. (2003). What we're learning about student engagement from NSSE: benchmarks for effective educational practices. Change 35, 24-32. doi: 10.1080/00091380309604090

Mai, L. W. (2005). A comparative study between UK and US: the student satisfaction in higher education and its influential factors. J. Market. Manag. 21, 859-878. doi: 10.1362/026725705774538471

Mathooko, F. M., and Ogutu, M. (2015). Porter's five competitive forces framework and other factors that influence the choice of response strategies adopted by public universities in Kenya. Int. J. Educ. Manag. 29, 334-354. doi: 10.1108/ijem-12-2013-0187

Milian, R. P., Davies, S., and Zarifa, D. (2016). Barriers to differentiation: applying organizational studies to Ontario higher education. Can. J. High. Educ. 46, 19-37. and perhaps most important, there is a need to better understand the concept of student satisfaction and how this is driven by the increasingly important economic consequences that studying in $\mathrm{HE}$ has for individual students. In short, student satisfaction is a key concept in the modern consumerist HE sector, but it is one that we still don't fully understand and don't know how to measure.

\section{AUTHOR CONTRIBUTIONS}

All authors listed have made substantial, direct and intellectual contributions to the work, and approved it for publication.

\section{FUNDING}

We acknowledge the support of the National Teaching Fellowship to CS.

Moores, E., and Reddy, P. (2012). No regrets? Measuring the career benefits of a psychology placement year. Assess. Eval. High. Educ. 37, 535-554. doi: 10.1080/02602938.2011.553668

Ramsden, P. (1991). A performance indicator of teaching quality in higher education: the course experience questionnaire. Stud. High. Educ. 16, 129-150. doi: 10.1080/03075079112331382944

Reddy, P., and Moores, E. (2012). Placement year academic benefit revisited: effects of demographics, prior achievement and degree programme. Teach. High. Educ. 17, 153-165. doi: 10.1080/13562517.2011.611873

Richardson, J. T., Slater, J. B., and Wilson, J. (2007). The national student survey: development, findings and implications. Stud. High. Educ. 32, 557-580. doi: 10.1080/03075070701573757

Scott, P. (1995). "State and society," in The Globalisation of Higher Education. Society for Research into Higher Education, ed P. Scott (Philadelphia, PA: OU Press).

Senior, C., Reddy, P. A., and Senior, R. (2014). The relationship between student employability and student engagement: working toward a more unified theory. Front. Educ. Psychol. 5:238. doi: 10.3389/fpsyg.2014. 00238

Tomlinson, M. (2008). 'The degree is not enough': students' perceptions of the role of higher education credentials for graduate work and employability. Br. J. Sociol. Educ. 29, 49-61. doi: 10.1080/01425690701 737457

Van de Ven, A. H. (2007). Engaged Scholarship: A Guide for Organizational and Social Research. Oxford, UK: Oxford University Press on Demand.

Wilson, K. L., Lizzio, A., and Ramsden, P. (1997). The development, validation and applications of the course experience questionnaire. Stud. High. Educ. 22, 33-53. doi: 10.1080/03075079712331381121

Yamada, R. (2013). "The present situation of quality enhancement in Japan," in Enhancing Quality in Higher Education: International Perspectives, eds R. Land and G. Gordon (Oxford, UK: Routledge), 213-224.

Yorke, M., Orr, S., and Blair, B. (2014). Hit by a perfect storm? Art and Design in the National Student Survey. Stud. High. Educ. 39, 1788-1810. doi: 10.1080/03075079.2013.806465

Conflict of Interest Statement: The authors declare that the research was conducted in the absence of any commercial or financial relationships that could be construed as a potential conflict of interest.

Copyright (c) 2017 Senior, Moores and Burgess. This is an open-access article distributed under the terms of the Creative Commons Attribution License (CC BY). The use, distribution or reproduction in other forums is permitted, provided the original author(s) or licensor are credited and that the original publication in this journal is cited, in accordance with accepted academic practice. No use, distribution or reproduction is permitted which does not comply with these terms. 